\title{
KNOWLEDGE MANAGEMENT SUPPORT FOR COOPERATIVE RESEARCH
}

\author{
Jun Ma and Matthias Hemmje
}

Fraunhofer-IPSI, Dolivostrasse 15, Darmstadt D-64293, Germany

\{jun,hemmje\}@ipsi.fhg.de,Tel:+49-6151-869-4884; +49-6151-869-844

\begin{abstract}
A prototype system designed for enhancing the cooperative research of groups and individuals in research institutes is provided. The main implementing techniques are introduced as well.
\end{abstract}

Keywords: knowledge management, knowledge filters, knowledge audit, knowledge reuse.

\section{INTRODUCTION}

A prototype system designed based on the research activities of Fraunhofer-IPSI for enhancing the cooperative research of groups and individuals is introduced. Compared with some known knowledge management $(\mathrm{KM})$ systems $[1,2,4-6]$, the design is human-centred and service-oriented, i.e. it is emphasized more to enhance working efficiency, decrease expense, avoid reinventing, reuse former working experience and strengthen co-research in innovative organizations by providing intelligent services. An outline of the main functions of the prototype system is shown in Fig. 1.

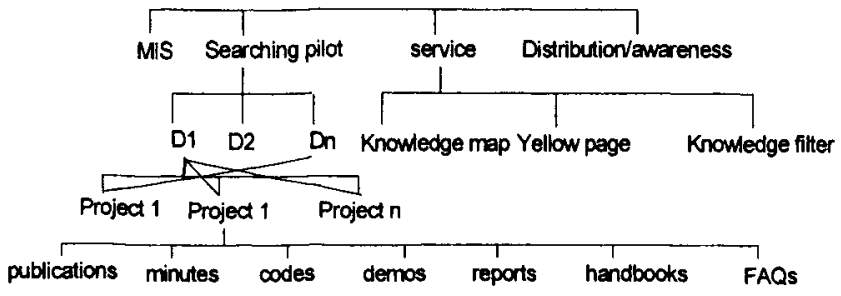

Figure 1. An outline of the prototype system. 


\section{THE MAIN IMPLEMENTATION TECHNIQUES}

The design and implementation of the system mainly utilizes the databases recording the profiles of employees and groups as well as the documents describing the best practices and the projects carried out before. A personal profile consists of research interests, the projects participated, the roles in these projects, education and training as well as publications. A group profile describes the members in the group and the synergy relations with other partners. The descriptions of the projects and best practices in the system are presented in meta-documents that are classified into several categories according to application domains denoted by $D 1, D 2, \ldots, D n$ and the types of documents shown in Fig. 1. These documents are organized in hierarchy based on the subclass-of relations defined on the categories. For each application domain, the learning materials is presented by a directed graph $G i(\mathrm{~V}, \mathrm{~A})$ without directed cycles, where $\mathrm{V}$ consists of the names of the learning materials; $\forall u, v \in \mathrm{V},\langle u, v>\in \mathrm{A}$ iff it is better for a learner to study $u$ before to study $v$. Furthermore the learning materials are classified as the elementary and the professional based on their contents.

Some techniques used in the project are now introduced below.

1. Knowledge audit: When one wants to participate a project related to a domain $D i$, based on his/her profile, e.g. the courses leaned, and the corresponding directed graph $G i$, the system helps him/her to find which knowledge is missing and how to schedule his/her study as well as recommends suitable learning materials to him/her.

2. Knowledge mapping: based on the profiles of individuals and groups, the system displays various relationships graphically, e.g., the technical and research synergy relations among individuals, groups and the partners inside and outside the institute. The detail information of the partners can be provided based on the yellow pages of the system as well.

3. Knowledge bases: The descriptions of the best practices and projects carried out before are presented in meta-documents, where meta-data are used to annotate the entities in a meta-document and the semantic relationships among the entities in the meta-document. An entity may be a demo, a literal report, and a piece of graphic and image. The semantic relationships among the entities are denoted in RDF and XML [8], while the documents corresponding to these entities may be dispersed across a number of servers.

4. Knowledge awareness/distribution: the system provides each group a shared file space that allows group members to upload documents and in the meantime informs others to access them. A shared calendar shows the schedules of group members in coming weeks. Based on the users' searching requirements and the personal profiles, e.g. publications, the roles in projects and the projects participated before, the system can also 
recommend the experts in some application areas within organizations and partners.

In the following talk, we describe a knowledge filter in detail because for the time being knowledge filtering is done mainly by human, e.g. accepting the consensus of experts [7]. Our algorithm is different from some known collaborative filters, e.g. the algorithms provided in [3], in terms of multievaluation factors with different weights are synthetically considered.

The main usage of the knowledge filter is to rank files based on the comments of experts or peers. Then the knowledge filter will generate a synthetic comment in order to identify whether files should be added into the knowledge bases or the databases or other file systems. The algorithm for knowledge filtering collects the comments of experts on the Internet and produces the synthetic comment based on fuzzy computation and statistics. The detail algorithm is described below.

Let $P$ represent a file, e.g., which is a proposal or business strategy. We design an $\mathrm{m} \times \mathrm{n}$ matrix to collect the comments for $\mathrm{P}$ from experts. Let $\mathrm{U}=$ $\left\{u_{1}, u_{2}, \ldots, u_{n}\right\}$, where $u_{i}, 1 \leq i \leq n$, is an evaluation factor, such as, originality, creativity, presentation, relativity and so on. The comment set $\mathrm{V}=$ $\left\{v_{1}, v_{2}, \ldots, v_{m}\right\}$, where $v_{i}$ is a comment, $1 \leq i \leq m$, e.g. "bad", "normal", "good" and "excellent". If an expert chooses a comment $v_{i}$ to evaluation factor $u_{j}$ for $\mathrm{P}$, then he/she gives a mark at the position $(i, j)$ of the table. Following algorithm gives a synthetic comment by fuzzy and statistic computation to determine whether the knowledge is good enough to be added to knowledge bases. The detail will be discussed below.

Algorithm (synthetic comment computation)

Input:

1. A file P;

2. The evaluation factor set $\mathrm{U}=\left\{u_{1}, u_{2}, \ldots, u_{n}\right\}$, where $u_{i}, 1 \leq i \leq n$, is an evaluation factor;

3. The comment set $\mathrm{V}=\left\{v_{1}, v_{2}, \ldots, v_{m}\right\}$, where $v_{i}$ is a comment, $1 \leq i \leq m$.

4. A fuzzy vector $\mathrm{X}=\left(x_{1} / u_{1}, x_{2} / u_{2}, \cdots, x_{n} / u_{n}\right)$, such that, $\sum_{1}^{n} x_{i}=1$. $x_{i} \geq 0, i=1,2, \ldots, n . \quad x_{i}$ represents the importance of the factor $u_{i}$ in the evaluation.

5. The experts' comments on $P$.

Output: A synthetic comment of $P$.

Step 1. After collecting the comments from all referees for a file $\mathrm{P}$, calculate following fuzzy sets:

$\left(\mu_{i 1} / v_{1}, \mu_{i 2} / v_{2}, \cdots, \mu_{i m} / v_{m}\right), 0 \leq \mu_{i j} \leq 1, i=1,2, \ldots n$;

$j=1,2, \ldots m$; Where $\mu_{i j}=$ (the number of persons who give $v_{j}$ to $u_{i}$ )/ (total number of referees) $\times 100 \%$. $\quad N$ fuzzy sets are represented in a matrix $E=\left(\mu_{i j}\right)_{n \times m}$. 
Step 2. Calculate following vector

$$
\begin{aligned}
& \left(y_{1} / v_{1}, y_{2} / v_{2}, \cdots, y_{m} / v_{m}\right) \text { by following formula. } \\
& y_{i}=\underset{i=1}{\vee}\left(x_{i} \wedge \mu_{i j}\right), j=1,2, \cdots m .
\end{aligned}
$$

Where $\vee$ and $\wedge$ represent a suitable operation, e.g. $\mathbf{a} \vee \mathbf{b}=$ $\max (a, b)$; and $a \wedge b=\min (a, b)$;

Step 3. If $y_{k}$ is of the maximum in set $\left\{y_{1}, y_{2}, \ldots y_{m}\right\}$, then output $v_{k}$ as the synthetic comment.

$\mathrm{P}$ will be added to knowledge base if $v_{k}$ is good enough. The principle of the algorithm can be stated as follows. We do the statistics computation in the step 2, where the percentage of the comment $v_{j}$ gotten in all given comments on evaluation factor $u_{i}$ is calculated, $1 \leq i \leq n$ and $1 \leq j \leq m$. In step 3 , based on the principle of fuzzy processing technique, a fuzzy vector $\mathrm{X}=$ $\left(x_{1} / u_{1}, x_{2} / u_{2}, \cdots, x_{n} / u_{n}\right)$ is used, where $x_{i}$ represents the importance of evaluation factor $u_{i}$ in the computation of a synthetic comment on a proposal $\mathrm{P} . \quad x_{i}$ is as a weight value in the calculation of the mathematics expectation of each $v_{j}, 1 \leq j \leq m$ also. Clearly the computation of mathematics expectation of $v_{i}, 1 \leq i \leq m$, considers both the comments from all referees and the importance of each evaluation factor. We choose the one with the maximum mathematics expectation among all comments as the final comment on P.

In this paper we want to show many functions of KM systems can be designed and implemented based on the descriptions of the profiles of employees and groups; knowledge bases can be organized based on the metadocuments that describe the best practice and projects in the system. We also introduce a design of a knowledge filter. We want to show a service-oriented $\mathrm{KM}$ system can provide a convenient environment for researchers in their daily work.

\section{References}

[1] Borghoff U.M., and Pareschi R., Information Technology for Knowledge Management, Springer-Verlag press, $1998,1-8$.

[2] Caldwell N.H.M., Clarkson P.J., Rodgers P.A. and Huxor A.P., Web-Based Knowledge Management for Distributed Design, IEEE Intelligent systems, May/June 2000, 40-47.

[3] Collaborative Filters, http://www.acm.org/chi95/Electronic/documents/papers.

[4] Kim K.H., and Paik S.K. Practical Experiences and Requirements on Workflow, Proceedings on Coordination Technology for Collaborative Applications, Wolfram Conen and G.Neumann (Eds), Singapore, Springer-Verlag press, 1997, pp145-160.

[5] Kappel G., Schott S.R. and Retschitzegger W., Proceedings on Coordination in Workflow Management Systems A Rule-Based Approach, Coordination Technology for Collaborative Applications, Eds. Wolfram Conen, Gr Neumann, Singapore, Springer-Verlag press, 1997, 100-119.

[6] Nonaka I. and Takeuchi H., The knowledge Creating Company: How Japanese Companies Create the Dynamics of Innovation, New York: Oxford Univ. Press, 1995

[7] O'Leary D.E, Enterprise knowledge management, IEEE on Computer, March, 1998, 54-61.

[8] RDF and XML, www.w3.org/TR. 